\title{
Article \\ Consensus Control of Leaderless and Leader-Following Coupled PDE-ODEs Modeled Multi-Agent Systems
}

\author{
Xu Ni ${ }^{1,+}$, Kejia Yi ${ }^{2,+}$, Yiming Jiang ${ }^{3,+}+\mathbb{C}$, Ancai Zhang ${ }^{4,+}$ and Chengdong Yang ${ }^{5, *,+}$ \\ 1 Faculty of Management and Economics, Kunming University of Science and Technology, \\ Kunming 650500, China; nixu2019@kust.edu.cn \\ 2 Systems Engineering Research Institute, China State Shipbuilding Corporation Limited, \\ Beijing 100094, China; seriyikejia2018@163.com \\ 3 School of Precision Instrument and Opto-electronics Engineering, Tianjin Univeristy, \\ Tianjin 300072, China; 1meanj@gmail.com \\ 4 School of Automation and Electrical Engineering, Linyi University, Linyi 276005, China; \\ zhangancai@lyu.edu.cn \\ 5 School of Information Science and Technology, Linyi University, Linyi 276005, China \\ * Correspondence: yangchengdong@lyu.edu.cn \\ + All authors contributed equally to this work.
}

check for updates

Citation: Ni, X.; Yi, K.; Jiang, Y.; Zhang, A.; Yang, C. Consensus Control of Leaderless and Leader-Following Coupled PDE-ODEs Modeled Multi-Agent Systems. Mathematics 2022, 10, 201. https://doi.org/10.3390/math 10020201

Academic Editors: Fang Liu and Qianyi Liu

Received: 13 December 2021

Accepted: 7 January 2022

Published: 10 January 2022

Publisher's Note: MDPI stays neutral with regard to jurisdictional claims in published maps and institutional affiliations.

Copyright: (C) 2022 by the authors. Licensee MDPI, Basel, Switzerland. This article is an open access article distributed under the terms and conditions of the Creative Commons Attribution (CC BY) license (https:// creativecommons.org/licenses/by/ $4.0 /)$.

\begin{abstract}
This paper discusses consensus control of nonlinear coupled parabolic PDE-ODE-based multi-agent systems (PDE-ODEMASs). First, a consensus controller of leaderless PDE-ODEMASs is designed. Based on a Lyapunov-based approach, coupling strengths are obtained for leaderless PDE-ODEMASs to achieve leaderless consensus. Furthermore, a consensus controller in the leaderfollowing PDE-ODEMAS is designed and the corresponding coupling strengths are obtained to ensure the leader-following consensus. Two examples show the effectiveness of the proposed methods.
\end{abstract}

Keywords: consensus; PDE-ODEs; MASs; leader-following; coupling strengths

\section{Introduction}

Consensus in multi-agent systems (MASs) is to achieve a common group objective when agents have different initial states [1-4]. It has received great attention in the past decade as a result from its wide applications in flocking of mobile robots [5], opinion dynamics in social networks [6], formation of unmanned vehicles [7-9], microgrid energy management [10], traffic flow [11], etc.

In a pioneering contribution, many important control methods were proposed for consensus of MASs, focusing on models based on ordinary differential equations [12-20]. Actually, there are many practical cases in nature and discipline fields with spatio-temporal characteristics, modeled by coupled partial differential equations (PDEs) [21-25]. Applied to overhead cranes [26], hormonal therapy [27], traffic flow [28], etc., another class of spatio-temporal models is based on coupled partial differential equations-ordinary differential equations (PDE-ODEs) [29-31]. Therefore, it is important to research consensus control of PDE-based coupled MASs (PDEMASs) or coupled PDE-ODE-based MASs (PDE-ODEMASs).

More recently, there have been many important results related to PDEMASs. Ref. [32] studied a distributed adaptive controller of uncertain leader-following parabolic PDEMASs; ref. [33] studied consensus control for parabolic and second-order hyperbolic PDEMASs; ref. [34] studied distributed P-type iterative learning for PDEMASs with time delay; refs. $[35,36]$ studied iterative learning control for PDEMASs without and with time delay; ref. [37] studied boundary control of 3-D PDEMASs with arbitrarily large boundary input delay; refs. [38,39] studied consensus and input constraint consensus of nonlinear PDEMASs using boundary control. However, consensus control for PDE-ODEMASs has not been addressed yet, which is a new challenge. 
Motivated by the above, this paper studies consensus control of nonlinear coupled parabolic PDE-ODEMASs with Neumann boundary conditions. First, dealing with the leaderless case, a consensus controller of leaderless PDE-ODEMASs is designed. The leaderless consensus error system is obtained and one Lyapunov functional candidate is given. Using Wirtinger's inequality and matrix properties, coupling strengths are obtained for leaderless PDE-ODEMASs to achieve cluster consensus. Furthermore, dealing with the leader-following case, a consensus controller of leader-following PDE-ODEMASs is designed. The leader-following consensus error system is obtained and another Lyapunov functional candidate is given. The corresponding coupling strengths are obtained to ensure leader-following consensus.

The remainder of this paper is organized as follows. The problem formulation is given in Section 2. Section 3 presents a consensus control design of the leaderless PDE-ODEMAS and Section 4 gives that of the leader-following PDE-ODEMAS. An example to illustrate the effectiveness of the proposed method is presented in Sections 5 and 6 offers some concluding remarks.

Notations: $\lambda_{\max }(\cdot), \lambda_{2}(\cdot)$ stand for the maximum eigenvalue and smallest nonzero eigenvalue of $\cdot$, respectively. $\otimes$ is a Kronecker product of matrices. The identity matrix of $n$ order is denoted by $I_{n}$. $\|\cdot\|$ denotes the Euclidean norm for vectors in $R^{n}$ or the induced 2-norm for matrices in $\boldsymbol{R}^{m \times n}$.

\section{Problem Formulation}

Consider a nonlinear PDE-ODEMAS as

$$
\begin{gathered}
\dot{x}_{i}(t)=f\left(x_{i}(t)\right)+\int_{0}^{1} w\left(y_{i}(\xi, t)\right) d \xi+u_{i}(t), \\
\begin{aligned}
\frac{\partial y_{i}(\xi, t)}{\partial t} & =\alpha \frac{\partial^{2} y_{i}(\xi, t)}{\partial \xi^{2}}+p\left(y_{i}(\xi, t)\right) \\
& +q\left(x_{i}(t)\right)+U_{i}(\xi, t)
\end{aligned}
\end{gathered}
$$

such that

$$
\begin{aligned}
& \left.\frac{\partial y_{i}(\xi, t)}{\partial \xi}\right|_{\xi=0}=0,\left.\frac{\partial y_{i}(\xi, t)}{\partial \xi}\right|_{\xi=1}=0, \\
& x_{i}(0)=x_{i}^{0}, y_{i}(\xi, 0)=y_{i}^{0}(\xi),
\end{aligned}
$$

where $(\xi, t) \in[0,1] \times[0, \infty)$, respectively, mean the spatial variable and time variable; $x_{i}(t), y_{i}(\xi, t) \in \mathbb{R}^{n}$ are the states; $u_{i}(t), U_{i}(\xi, t) \in \mathbb{R}^{n}$ are the control inputs; $x_{i}^{0}, y_{i}^{0}(\xi)$ are bounded and $y_{i}^{0}(\xi)$ is continuous; $\alpha$ is a positive scalar; $i \in\{1,2, \cdots, N\}$; and $f(\cdot), w(\cdot), p(\cdot), q(\cdot) \in \mathbb{R}^{n}$ are sufficiently smooth nonlinear functions.

Define consensus error $e_{i}(t) \triangleq x_{i}(t)-\frac{1}{N} \sum_{j=1}^{N} x_{j}(t)$ and $\varepsilon_{i}(\xi, t) \triangleq y_{i}(\xi, t)-\frac{1}{N} \sum_{j=1}^{N} y_{j}(\xi, t)$.

Definition 1. For the leaderless PDE-ODEMAS (1), (2) with any initial conditions, if

$$
\lim _{t \rightarrow \infty} e_{i}(t) \rightarrow 0, \lim _{t \rightarrow \infty} \varepsilon_{i}(\xi, t) \rightarrow 0,
$$

for any $i \in\{1,2, \cdots, N\}$, then the leaderless PDE-ODEMAS (1), (2) achieves consensus.

Lemma 1 ([40]). Let $\kappa$ be a differentiable function with $\kappa(0)=0$ and $\kappa(1)=0$, then

$$
\int_{0}^{1} \kappa^{T}(s) \kappa(s) d s \leq \pi^{-2} \int_{0}^{1} \dot{\kappa}^{T}(s) \dot{\kappa}(s) d s .
$$

Lemma $2([41])$. For an undirected connected graph with Laplacian matrix $L$, and $x \in \mathbb{R}^{n}$ such that $1_{N}^{T} x=0$, then

$$
\lambda_{2}(L) x^{T} x \leq x^{T} L x
$$


If Laplacian matrix $L \in \mathbb{R}^{N \times N}$ is symmetric, then $0=\lambda_{1}(\cdot)<\lambda_{2}(\cdot) \leq \cdots \leq$ $\lambda_{N}(\cdot)$. The smallest nonzero eigenvalue of $\lambda_{2}(\cdot)$ is known as the algebraic connectivity of graphs [41].

Assumption 1. Assume $f(\cdot), p(\cdot), q(\cdot), w(\cdot)$ satisfy the Lipschitz condition, i.e., for any $v_{1}$ and $v_{2} \in \mathbb{R}^{n}$, there exist scalars $\gamma_{1}, \gamma_{2}, \gamma_{3}, \gamma_{4}>0$ such that

$$
\begin{aligned}
& \left|f\left(v_{1}\right)-f\left(v_{2}\right)\right| \leq \gamma_{1}\left|v_{1}-v_{2}\right|, \\
& \left|p\left(v_{1}\right)-p\left(v_{2}\right)\right| \leq \gamma_{2}\left|v_{1}-v_{2}\right|, \\
& \left|q\left(v_{1}\right)-q\left(v_{2}\right)\right| \leq \gamma_{3}\left|v_{1}-v_{2}\right|, \\
& \left|w\left(v_{1}\right)-w\left(v_{2}\right)\right| \leq \gamma_{4}\left|v_{1}-v_{2}\right| .
\end{aligned}
$$

\section{Consensus Control of the Leaderless PDE-ODEMAS}

To achieve consensus of the leaderless PDE-ODEMAS (1), the consensus controller is designed as:

$$
\begin{aligned}
& u_{i}(t)=d \sum_{j=1}^{N} a_{i j}\left(x_{j}(t)-x_{i}(t)\right), \\
& U_{i}(\xi, t)=k \sum_{j=1}^{N} b_{i j}\left(y_{j}(\xi, t)-y_{i}(\xi, t)\right),
\end{aligned}
$$

where $d$ and $k$ are the coupling strengths to be determined, $i \in\{1,2, \cdots, N\}$. Assume that the topological structure $A=\left(a_{i j}\right)_{N \times N}$ is defined as: $a_{i j}=a_{j i}>0(i \neq j)$ if the agent $i$ connects to $j$, otherwise $a_{i j}=0(i \neq j) ; a_{i i}=0$. The topological structure $B=\left(b_{i j}\right)_{N \times N}$ is defined the same as $A$.

The consensus error system can be obtained from (1), (2), and (7) that

$$
\begin{aligned}
\dot{e}_{i}(t)= & f\left(x_{i}(t)\right)-\frac{1}{N} \sum_{j=1}^{N} f\left(x_{j}(t)\right)+\int_{0}^{1} w\left(y_{i}(\xi, t)\right) d \xi-\frac{1}{N} \sum_{j=1}^{N} \int_{0}^{1} w\left(y_{j}(\xi, t)\right) d \xi \\
+ & d \sum_{j=1}^{N} a_{i j}\left(x_{j}(t)-x_{i}(t)\right), \\
\frac{\partial \varepsilon_{i}(\xi, t)}{\partial t}= & \alpha \frac{\partial^{2} \varepsilon_{i}(\xi, t)}{\partial \xi^{2}}+p\left(y_{i}(\xi, t)\right)-\frac{1}{N} \sum_{j=1}^{N} p\left(y_{j}(\xi, t)\right)+q\left(x_{i}(t)\right) \\
& -\frac{1}{N} \sum_{j=1}^{N} q\left(x_{j}(t)\right)+k \sum_{j=1}^{N} b_{i j}\left(y_{j}(\xi, t)-y_{i}(\xi, t)\right),
\end{aligned}
$$

such that

$$
\begin{aligned}
& \left.\frac{\partial \varepsilon_{i}(\xi, t)}{\partial \xi}\right|_{\xi=0}=0,\left.\frac{\partial \varepsilon_{i}(\xi, t)}{\partial \xi}\right|_{\xi=1}=0, \\
& e_{i}(0)=e_{i}^{0}(\xi), \varepsilon_{i}(\xi, 0)=\varepsilon_{i}^{0}(\xi),
\end{aligned}
$$

where $e_{i}^{0} \triangleq x_{i}^{0}-\frac{1}{N} \sum_{j=1}^{N} x_{j}^{0}$ and $\varepsilon_{i}^{0}(\xi) \triangleq y_{i}^{0}(\xi)-\frac{1}{N} \sum_{j=1}^{N} y_{j}^{0}(\xi)$.

Theorem 1. Under Assumption 1, assume the graphs A and B are connected. Using the controller (7), the leaderless PDE-ODEMAS (1), (2) achieves consensus if

$$
\begin{aligned}
& d>\frac{\gamma_{1}+\frac{1}{2} \gamma_{3}^{2}+\frac{1}{2}}{\lambda_{2}\left(L_{a}\right)} \\
& k>\max \left\{\frac{\gamma_{2}+\frac{1}{2} \gamma_{4}^{2}+\frac{1}{2}-\alpha \pi^{2}}{\lambda_{2}\left(L_{b}\right)}, 0\right\} .
\end{aligned}
$$


Proof. Consider the following Lyapunov function as

$$
V_{1}(t)=\frac{1}{2} \sum_{i=1}^{N} e_{i}^{T}(t) e_{i}(t)+\frac{1}{2} \sum_{i=1}^{N} \int_{0}^{1} \varepsilon_{i}^{T}(\xi, t) \varepsilon_{i}(\xi, t) d \xi .
$$

We have

$$
\begin{aligned}
\dot{V}_{1}(t)= & \sum_{i=1}^{N} e_{i}^{T}(t) \dot{e}_{i}(t)+\sum_{i=1}^{N} \int_{0}^{1} \varepsilon_{i}^{T}(\xi, t) \frac{\partial \varepsilon_{i}(\xi, t)}{\partial t} d \xi \\
= & \sum_{i=1}^{N} e_{i}^{T}(t)\left[f\left(x_{i}(t)\right)-\frac{1}{N} \sum_{j=1}^{N} f\left(x_{j}(t)\right)\right] \\
& +\sum_{i=1}^{N} e_{i}^{T}(t)\left[\int_{0}^{1} w\left(y_{i}(\xi, t)\right) d \xi-\frac{1}{N} \sum_{j=1}^{N} \int_{0}^{1} w\left(y_{j}(\xi, t)\right) d \xi\right] \\
& +\sum_{i=1}^{N} e_{i}^{T}(t) d \sum_{j=1}^{N} a_{i j}\left(e_{j}(t)-e_{i}(t)\right)+\sum_{i=1}^{N} \int_{0}^{1} \varepsilon_{i}^{T}(\xi, t) \Theta \frac{\partial^{2} \varepsilon_{i}(\xi, t)}{\partial \xi^{2}} d \xi \\
& +\sum_{i=1}^{N} \int_{0}^{1} \varepsilon_{i}^{T}(\xi, t)\left(p\left(y_{i}(\xi, t)\right)-\frac{1}{N} \sum_{j=1}^{N} p\left(y_{j}(\xi, t)\right)\right) d \xi \\
& +\sum_{i=1}^{N} \int_{0}^{1} \varepsilon_{i}^{T}(\xi, t)\left(q\left(x_{i}(t)\right)-\frac{1}{N} \sum_{j=1}^{N} q\left(x_{j}(t)\right)\right) d \xi \\
& +\sum_{i=1}^{N} \int_{0}^{1} \varepsilon_{i}^{T}(\xi, t) k \sum_{j=1}^{N} b_{i j}\left(\varepsilon_{j}(\xi, t)-\varepsilon_{i}(\xi, t)\right) d \xi .
\end{aligned}
$$

According to the matrix property,

$$
\begin{aligned}
& \sum_{i=1}^{N} e_{i}^{T}(t) d \sum_{j=1}^{N} a_{i j}\left(e_{j}(t)-e_{i}(t)\right) \\
= & -d e^{T}(t)\left(L_{a} \otimes I_{n}\right) e(t) \\
\leq & -d \lambda_{2}\left(L_{a}\right) e^{T}(t) e(t)
\end{aligned}
$$

and

$$
\begin{aligned}
& \sum_{i=1}^{N} \int_{0}^{1} \varepsilon_{i}^{T}(\xi, t) k \sum_{j=1}^{N} b_{i j}\left(\varepsilon_{i}(\xi, t)-\varepsilon_{j}(\xi, t)\right) d \xi \\
= & -k \int_{0}^{1} \varepsilon^{T}(\xi, t)\left(L_{b} \otimes I_{n}\right) \varepsilon(\xi, t) d \xi \\
\leq & -k \lambda_{2}\left(L_{b}\right) \int_{0}^{1} \varepsilon^{T}(\xi, t) \varepsilon(\xi, t) d \xi
\end{aligned}
$$

where $\lambda_{2}(\cdot)$ denotes the smallest nonzero eigenvalue of $\cdot L_{a, i j}=-a_{i j}$ when $i \neq j, L_{a, i i}=$ $\sum_{j=1}^{N} a_{i j}, L_{b, i j}=-b_{i j}$ when $i \neq j, L_{b, i i}=\sum_{j=1}^{N} b_{i j}$. Therefore, $L_{a}, L_{b}$ are Laplacian matrices. Using Lemma 1, for $\alpha>0$, 


$$
\begin{aligned}
& \int_{0}^{1} \sum_{i=1}^{N} \varepsilon_{i}^{T}(\xi, t) \alpha \varepsilon_{i, \zeta \xi}(\xi, t) d \xi \\
= & -\alpha \int_{0}^{1} \varepsilon_{\xi}^{T}(\xi, t) \varepsilon_{\xi}(\xi, t) d \xi \\
\leq & -\alpha \pi^{2} \int_{0}^{1} \varepsilon^{T}(\xi, t) \varepsilon(\xi, t) d \xi .
\end{aligned}
$$

Using Assumption 1, owing to $\sum_{i=1}^{N} e_{i}^{T}(t)\left(f\left(\frac{1}{N} \sum_{j=1}^{N} x_{j}(t)\right)-\frac{1}{N} \sum_{j=1}^{N} f\left(x_{j}(t)\right)\right)=0$ and $\sum_{i=1}^{N} \varepsilon_{i}^{T}(\xi, t)\left(f\left(\frac{1}{N} \sum_{j=1}^{N} p\left(y_{i}(\xi, t)\right)\right)-\frac{1}{N} \sum_{j=1}^{N} p\left(y_{i}(\xi, t)\right)=0\right.$, we have

$$
\sum_{i=1}^{N} e_{i}^{T}(t)\left(f\left(x_{i}(t)\right)-\frac{1}{N} \sum_{j=1}^{N} f\left(x_{j}(t)\right)\right) \leq \gamma_{1} \sum_{i=1}^{N} e_{i}^{2}(t)
$$

and

$$
\sum_{i=1}^{N} \int_{0}^{1} \varepsilon_{i}^{T}(\xi, t)\left(p\left(y_{i}(\xi, t)\right)-\frac{1}{N} \sum_{j=1}^{N} p\left(y_{j}(\xi, t)\right)\right) d \xi \leq \gamma_{2} \int_{0}^{1} \varepsilon_{i}^{2}(\xi, t) d \xi .
$$

In the same way,

$$
\begin{aligned}
& \sum_{i=1}^{N} \int_{0}^{1} \varepsilon_{i}^{T}(\xi, t)\left(q\left(x_{i}(t)\right)-\frac{1}{N} \sum_{j=1}^{N} q\left(x_{j}(t)\right)\right) d \xi \\
= & \sum_{i=1}^{N} \int_{0}^{1} \varepsilon_{i}^{T}(\xi, t)\left(q\left(x_{i}(t)\right)-q\left(\frac{1}{N} \sum_{j=1}^{N} x_{j}(t)\right)\right) d \xi \\
\leq & \frac{1}{2} \sum_{i=1}^{N} \int_{0}^{1} \varepsilon_{i}^{T}(\xi, t) \varepsilon_{i}(\xi, t) d \xi+\frac{1}{2} \sum_{i=1}^{N} \int_{0}^{1}\left(q\left(x_{i}(t)\right)-q\left(\frac{1}{N} \sum_{j=1}^{N} x_{j}(t)\right)\right)^{2} d \xi \\
\leq & \frac{1}{2} \sum_{i=1}^{N} \int_{0}^{1} \varepsilon_{i}^{T}(\xi, t) \varepsilon_{i}(\xi, t) d \xi+\frac{1}{2} \gamma_{3}^{2} \sum_{i=1}^{N} e_{i}^{2}(t),
\end{aligned}
$$

and

$$
\begin{aligned}
& \sum_{i=1}^{N} e_{i}^{T}(t) \int_{0}^{1}\left(w\left(y_{i}(\xi, t)\right)-\frac{1}{N} \sum_{j=1}^{N} w\left(y_{j}(\xi, t)\right)\right) d \xi \\
= & \sum_{i=1}^{N} e_{i}^{T}(t) \int_{0}^{1}\left(w\left(y_{i}(\xi, t)\right)-w\left(\frac{1}{N} \sum_{j=1}^{N} y_{j}(\xi, t)\right)\right) d \xi \\
\leq & \frac{1}{2} \sum_{i=1}^{N}\left[e_{i}^{T}(t) e_{i}(t) d \xi+\int_{0}^{1}\left(w\left(y_{i}(\xi, t)\right)-w\left(\frac{1}{N} \sum_{j=1}^{N} y_{j}(\xi, t)\right)\right)^{2} d \xi\right] \\
\leq & \frac{1}{2} \sum_{i=1}^{N} e_{i}^{T}(t) e_{i}(t)+\frac{1}{2} \sum_{i=1}^{N} \gamma_{4}^{2} \int_{0}^{1} \varepsilon_{i}^{2}(\xi, t) d \xi .
\end{aligned}
$$

Substituting (13)-(19) into (12),

$$
\begin{aligned}
\dot{V}_{1}(t) & \leq-\rho_{1} e^{T}(t) e(t)-\rho_{2} \int_{0}^{1} \varepsilon^{2}(\xi, t) d \xi \\
& \leq-2 \rho V(t),
\end{aligned}
$$


where $e \triangleq\left[e_{1}^{T}, e_{2}^{T}, \cdots, e_{N}^{T}\right]^{T}, \varepsilon \triangleq\left[\varepsilon_{1}^{T}, \varepsilon_{2}^{T}, \cdots, \varepsilon_{N}^{T}\right]^{T}, \rho_{1} \triangleq-\gamma_{1}-\frac{1}{2} \gamma_{3}^{2}-\frac{1}{2}+d \lambda_{2}\left(L_{a}\right), \rho_{2} \triangleq$ $-\gamma_{2}-\frac{1}{2} \gamma_{4}^{2}-\frac{1}{2}+\alpha \pi^{2}+k \lambda_{2}\left(L_{b}\right)$, and $\rho \triangleq \min \left\{\rho_{1}, \rho_{2}\right\}$.

Taking $d$ and $k$ as (10) yields,

$$
\rho_{1}>0, \rho_{2}>0
$$

It follows from (20) and (21) that $V_{1}(t) \leq V_{1}(0) \exp \{-2 \rho t\}$, which implies $e_{i}(t) \rightarrow 0$ and $\varepsilon_{i}(\xi, t) \rightarrow 0$ as $t \rightarrow \infty$ This completes the proof.

\section{Consensus Control of the Leader-Following PDE-ODEMAS}

The leader agent is supposed to be

$$
\begin{aligned}
& \dot{x}_{0}(t)=f\left(x_{0}(t)\right)+\int_{0}^{1} w\left(y_{0}(\xi, t)\right) d \xi, \\
& \frac{\partial y_{0}(\xi, t)}{\partial t}=\alpha \frac{\partial^{2} y_{0}(\xi, t)}{\partial \xi^{2}}+p\left(y_{0}(\xi, t)\right)+q\left(x_{0}(t)\right),
\end{aligned}
$$

such that

$$
\begin{aligned}
& \left.\frac{\partial y_{0}(\xi, t)}{\partial \xi}\right|_{\xi=0}=0,\left.\frac{\partial y_{0}(\xi, t)}{\partial \xi}\right|_{\xi=1}=0, \\
& x_{0}(0)=x_{0}^{0}, y_{0}(\xi, 0)=y_{0}^{0}(\xi),
\end{aligned}
$$

where $x_{0}^{0}, y_{0}^{0}(\xi)$ are bounded and $y_{0}^{0}(\xi)$ is continuous.

The leader-following consensus controller is designed as:

$$
\begin{aligned}
& u_{i}(t)=d\left[\sum_{j=1}^{N} a_{i j}\left(x_{j}(t)-x_{i}(t)\right)+\delta_{i}\left(x_{0}(t)-x_{i}(t)\right)\right], \\
& U_{i}(\xi, t)=k\left[\sum_{j=1}^{N} b_{i j}\left(y_{j}(\xi, t)-y_{i}(\xi, t)\right)+\rho_{i}\left(y_{0}(\xi, t)-y_{i}(\xi, t)\right)\right],
\end{aligned}
$$

where $\delta_{i}>0$ if $x_{i}$ can obtain the information of $x_{0}$; otherwise, $\delta_{i}=0$; and $\rho_{i}>0$ if $y_{i}$ can obtain the information of $y_{0}$; otherwise, $\rho_{i}=0$.

Let $\tilde{e}_{i}(t)=x_{i}(t)-x_{0}(t)$ and $\tilde{\varepsilon}_{i}(\xi, t)=y_{i}(\xi, t)-y_{0}(\xi, t)$. The leader-following consensus error system is obtained as

$$
\begin{aligned}
\dot{\tilde{e}}_{i}(t)= & f\left(x_{i}(t)\right)-f\left(x_{0}(t)\right)+\int_{0}^{1} w\left(y_{i}(\xi, t)\right) d \xi \\
- & \int_{0}^{1} w\left(y_{0}(\xi, t)\right) d \xi-d \sum_{j=1}^{N} g_{i j} \tilde{e}_{j}(t), \\
\frac{\partial \tilde{\varepsilon}_{i}(\xi, t)}{\partial t}= & \alpha \frac{\partial^{2} \tilde{\varepsilon}_{i}(\xi, t)}{\partial \xi^{2}}+p\left(y_{i}(\xi, t)\right)-p\left(y_{0}(\xi, t)\right) \\
& +q\left(x_{i}(t)\right)-q\left(x_{0}(t)\right) \\
& -k \sum_{j=1}^{N} h_{i j} \tilde{\varepsilon}_{j}(\xi, t),
\end{aligned}
$$

such that

$$
\begin{aligned}
& \left.\frac{\partial \tilde{\varepsilon}_{i}(\xi, t)}{\partial \xi}\right|_{\xi=0}=0,\left.\frac{\partial \tilde{\varepsilon}_{i}(\xi, t)}{\partial \xi}\right|_{\xi=1}=0, \\
& \tilde{e}_{i}(0)=\tilde{e}_{i}^{0}(\xi), \tilde{\varepsilon}_{i}(\xi, 0)=\tilde{\varepsilon}_{i}^{0}(\xi),
\end{aligned}
$$


where $G=\left[g_{i j}\right]=L_{A}+\operatorname{diag}\left\{\delta_{i}\right\}, H=\left[h_{i j}\right]=L_{B}+\operatorname{diag}\left\{\rho_{i}\right\}, \tilde{e}_{i}^{0} \triangleq x_{i}^{0}-x_{0}^{0}$ and $\tilde{\varepsilon}_{i}^{0}(\xi) \triangleq$ $y_{i}^{0}(\xi)-y_{0}^{0}(\xi)$.

Definition 2. For the leader-following PDE-ODEMAS (22), (23) with any initial conditions, if

$$
\lim _{t \rightarrow \infty} \tilde{e}_{i}(t) \rightarrow 0, \lim _{t \rightarrow \infty}\left\|\tilde{\varepsilon}_{i}(\xi, t)\right\| \rightarrow 0,
$$

for any $i \in\{1,2, \cdots, N\}$, then the leader-following PDE-ODEMAS (22), (23) achieves consensus.

Theorem 2. Under Assumption 1, assume the graphs A and B are connected. Using the controller (26), the leader-following PDE-ODEMAS (1) achieves consensus if

$$
\begin{aligned}
& d>\frac{\gamma_{1}+\frac{1}{2} \gamma_{3}^{2}+\frac{1}{2}}{\lambda_{\min }(G)} \\
& k>\max \left\{\frac{\gamma_{2}+\frac{1}{2} \gamma_{4}^{2}+\frac{1}{2}-\alpha \pi^{2}}{\lambda_{\min }(H)}, 0\right\} .
\end{aligned}
$$

Proof. Consider the Lyapunov functional candidate as

$$
V_{2}(t)=\frac{1}{2} \sum_{i=1}^{N} \tilde{e}_{i}^{T}(t) \tilde{e}_{i}(t)+\frac{1}{2} \sum_{i=1}^{N} \int_{0}^{1} \tilde{\varepsilon}_{i}^{T}(\xi, t) \tilde{\varepsilon}_{i}(\xi, t) d \xi .
$$

One has

$$
\begin{aligned}
\dot{V}_{2}(t)= & \sum_{i=1}^{N} \tilde{e}_{i}^{T}(t) \dot{\tilde{e}}_{i}(t)+\sum_{i=1}^{N} \int_{0}^{1} \tilde{\varepsilon}_{i}^{T}(\xi, t) \frac{\partial \tilde{\varepsilon}_{i}(\xi, t)}{\partial t} d \xi \\
= & \sum_{i=1}^{N} \tilde{e}_{i}^{T}(t)\left(f\left(x_{i}(t)\right)-f\left(x_{0}(t)\right)\right) \\
& +\sum_{i=1}^{N} \tilde{e}_{i}^{T}(t)\left[\int_{0}^{1} w\left(y_{i}(\xi, t)\right) d \xi-\int_{0}^{1} w\left(y_{0}(\xi, t)\right) d \xi\right] \\
& -\sum_{i=1}^{N} \tilde{e}_{i}^{T}(t) d \sum_{j=1}^{N} g_{i j} \tilde{e}_{j}(t) \\
& +\sum_{i=1}^{N} \int_{0}^{1} \tilde{\varepsilon}_{i}^{T}(\xi, t) \alpha \frac{\partial^{2} \tilde{\varepsilon}_{i}(\xi, t)}{\partial \xi^{2}} d \xi \\
& +\sum_{i=1}^{N} \int_{0}^{1} \tilde{\varepsilon}_{i}^{T}(\xi, t)\left(p\left(y_{i}(\xi, t)\right)-p\left(y_{0}(\xi, t)\right)\right) d \xi \\
& +\sum_{i=1}^{N} \int_{0}^{1} \tilde{\varepsilon}_{i}^{T}(\xi, t)\left(q\left(x_{i}(t)\right)-q\left(x_{0}(t)\right)\right) d \xi \\
& -\sum_{i=1}^{N} \int_{0}^{1} \tilde{\varepsilon}_{i}^{T}(\xi, t) k \sum_{j=1}^{N} h_{i j} \tilde{\varepsilon}_{j}(\xi, t) d \xi .
\end{aligned}
$$

Since $G$ and $H$ are symmetric positive definite matrices,

$$
\begin{aligned}
& -\sum_{i=1}^{N} \tilde{e}_{i}^{T}(t) d \sum_{j=1}^{N} g_{i j}\left(\tilde{e}_{j}(t)\right) \\
= & -d \tilde{e}^{T}(t)\left(G \otimes I_{n}\right) \tilde{e}(t) \\
\leq & -d \lambda_{\min }(G) \tilde{e}^{T}(t) \tilde{e}(t),
\end{aligned}
$$


and

$$
\begin{aligned}
& \sum_{i=1}^{N} \int_{0}^{1} \tilde{\varepsilon}_{i}^{T}(\xi, t) k \sum_{j=1}^{N} h_{i j} \tilde{\varepsilon}_{j}(\xi, t) d \xi \\
= & -k \int_{0}^{1} \tilde{\varepsilon}^{T}(\xi, t)\left(H \otimes I_{n}\right) \tilde{\varepsilon}(\xi, t) d \xi \\
\leq & -k \lambda_{\min }(H) \int_{0}^{1} \tilde{\varepsilon}^{T}(\xi, t) \tilde{\varepsilon}(\xi, t) d \xi,
\end{aligned}
$$

where $\tilde{e} \triangleq\left[\tilde{e}_{1}^{T}, \tilde{e}_{2}^{T}, \cdots, \tilde{e}_{N}^{T}\right]^{T}, \tilde{\varepsilon} \triangleq\left[\tilde{\varepsilon}_{1}^{T}, \tilde{\varepsilon}_{2}^{T}, \cdots, \tilde{\varepsilon}_{N}^{T}\right]^{T}, \lambda_{\min }(\cdot)$ denotes the smallest nonzero eigenvalue and $G, H$ are symmetric positive definite matrices.

Considering (13)-(19), and substituting (31)-(32) into (30),

$$
\begin{aligned}
\dot{V}_{2}(t) \leq & \left(\gamma_{1}+\frac{1}{2} \gamma_{3}^{2}+\frac{1}{2}-d \lambda_{\min }(G)\right) \tilde{e}^{T}(t) \tilde{e}(t)+\left(\gamma_{2}+\frac{1}{2} \gamma_{4}^{2}\right. \\
& \left.+\frac{1}{2}-\alpha \pi^{2}-k \lambda_{\min }(H)\right) \int_{0}^{1} \tilde{\varepsilon}^{T}(\xi, t) \tilde{\varepsilon}(\xi, t) d \xi .
\end{aligned}
$$

In a similar way to the analysis in Theorem 1, the proof can be completed.

Remark 1. Many papers have investigated stabilization control methods for PDE-ODE systems [29-31,42], while this paper investigates consensus control for PDE-ODE-based MASs, considering control based on coupling.

Remark 2. Many significant results were obtained for consensus control modeled by PDEMASs [32-39]. Different from PDEMASs, this paper investigates consensus control methods for PDE-ODEMASs, as well as considering leaderless and leader-following models.

\section{Numerical Simulation}

Example 1. Consider the leaderless PDE-ODEMAS (1) and (2) with coefficients as

$$
\begin{aligned}
& \alpha=0.8, f(\cdot)=w(\cdot)=p(\cdot)=q(\cdot)=\tanh (\cdot), \\
& a_{i j}=b_{i j}=1, \text { and } i \neq j, \text { for } i, j=1,2,3,4, \\
& n=2,
\end{aligned}
$$

and with random initial conditions.

It is obvious that $f(\cdot), p(\cdot), q(\cdot)$, and $w(\cdot)$ satisfy the Lipschitz condition with $\gamma_{1}=\gamma_{2}=$ $\gamma_{3}=\gamma_{4}=1$.

With Theorem 1, according to (10), $d>0.50$ and $k>0$ are obtained. Therefore, we take $d=0.51$ and $k=0.01$. It can be seen in Figures 1 and 2 that the leaderless PDE-ODEMAS achieves consensus with control gains $d=0.51$ and $k=0.01$.

From another point of view, $d=0.49$ and $k=0$ do not satisfy (10). It can be seen in Figures 3 and 4 that the leaderless PDE-ODEMAS cannot achieve consensus with control gains $d=0.49$ and $k=0$. 


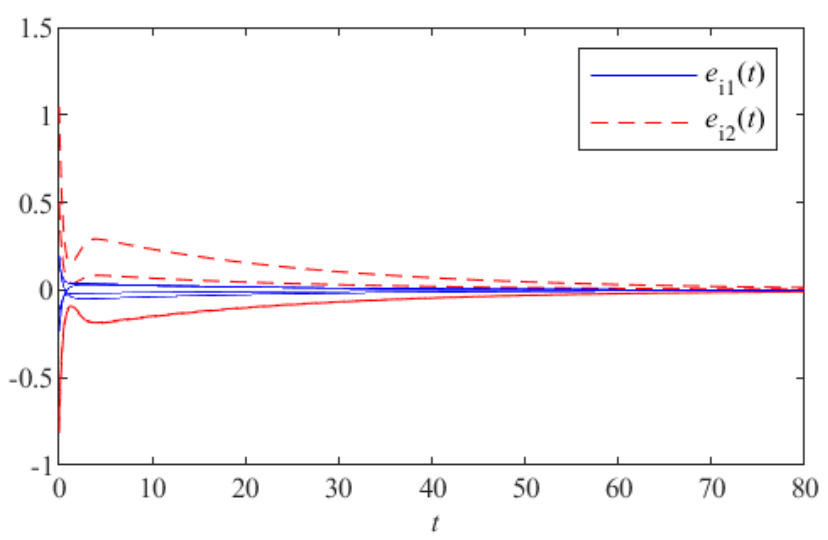

Figure 1. $e_{i}(t)$ withthe control gains $d=0.51$ and $k=0.01$ in Example 1 .
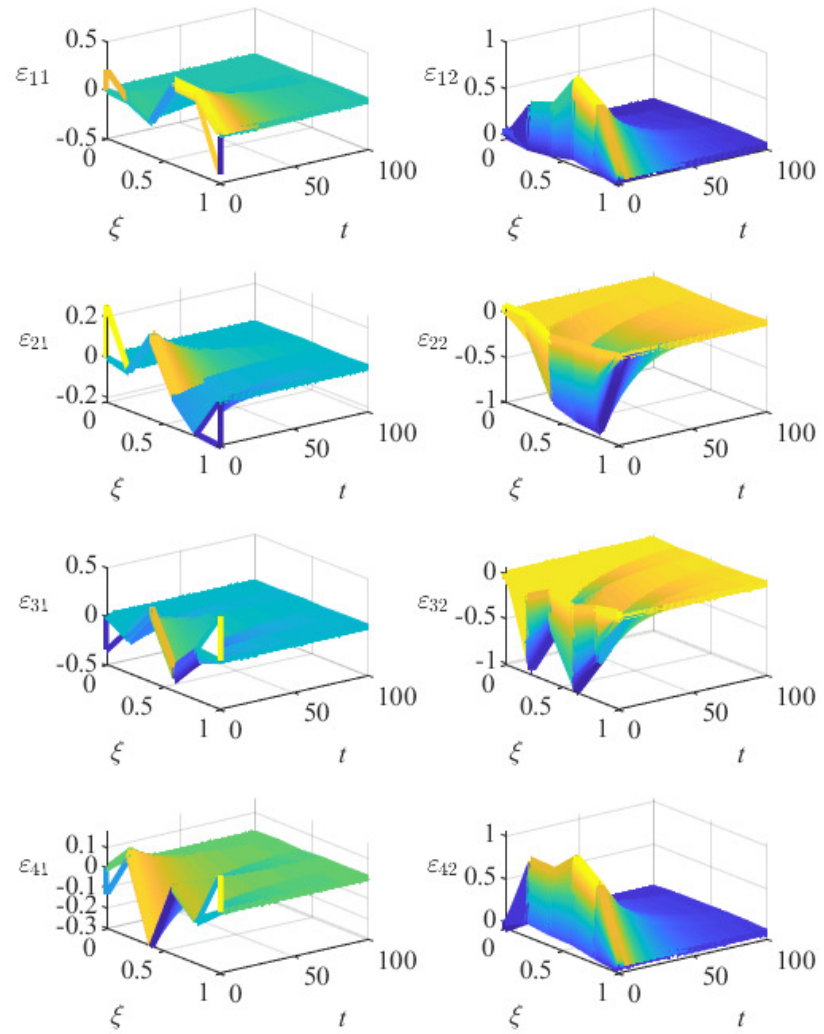

Figure 2. $\varepsilon_{i}(\xi, t)$ with the control gains $d=0.51$ and $k=0.01$ in Example 1 .

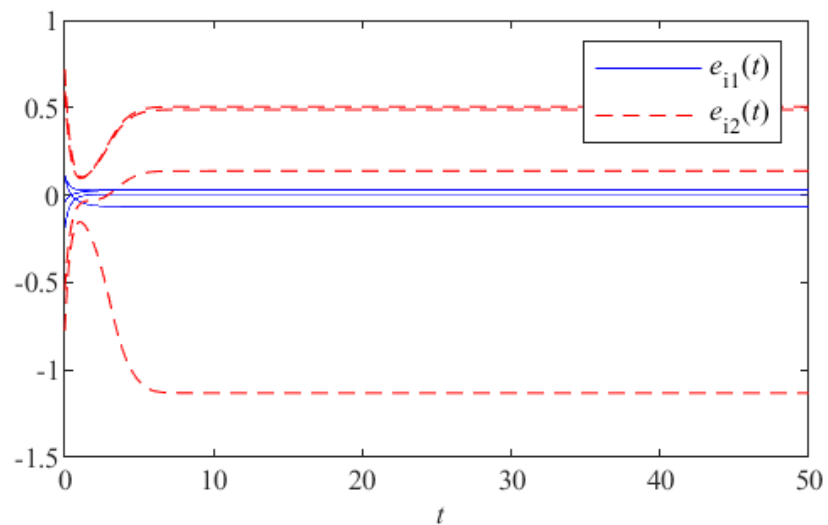

Figure 3. $e_{i}(t)$ with the control gains $d=0.49$ and $k=0$ in Example 1 . 

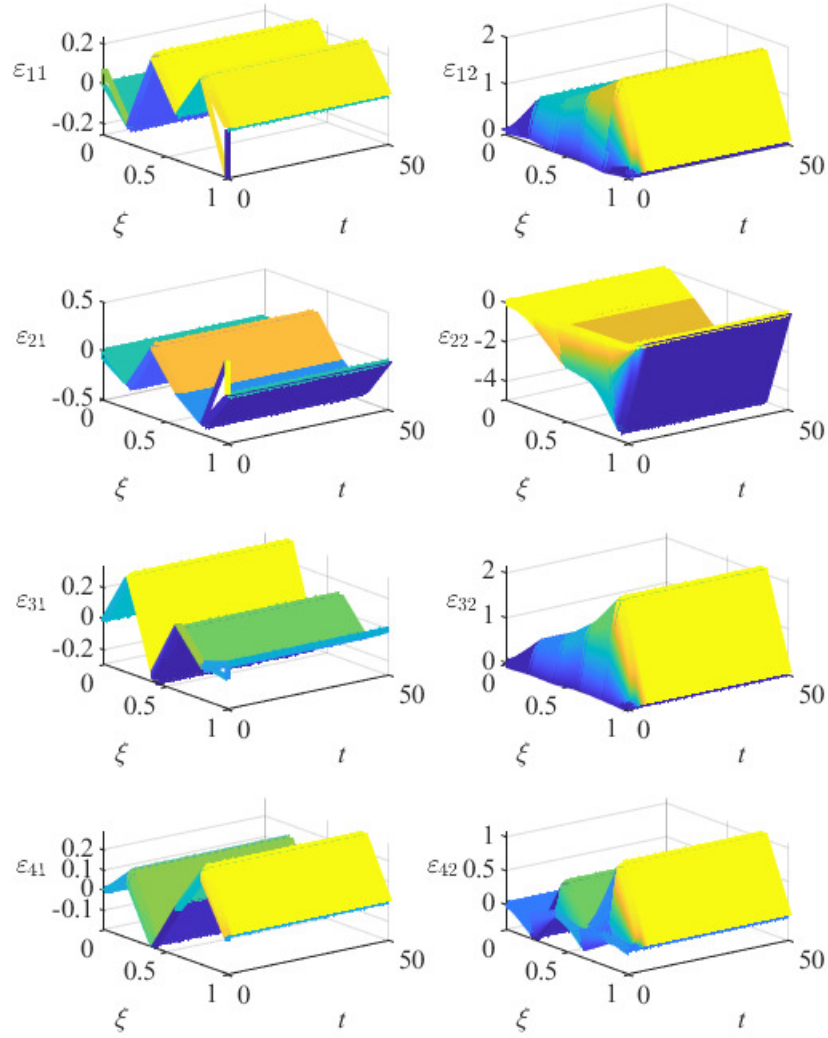

Figure 4. $\varepsilon_{i}(\xi, t)$ with the control gains $d=0.49$ and $k=0$ in Example 1.

Example 2. Consider a nonlinear leader-following PDE-ODEMAS composed of 1 leader agent (22) and (23) and 4 following agents (1) and (2) with coefficients the same as Example 1. In the same way, $\gamma_{1}=\gamma_{2}=\gamma_{3}=\gamma_{4}=1$ are obtained. Choose $\delta_{i}=\rho_{i}=1$. With Theorem 2, according to (28), $d>2.0$ and $k>0$ are obtained. Therefore, we take $d=2.1$ and $k=0.1$. It can be seen in Figures 5 and 6 that the leader-following PDE-ODEMAS achieves consensus.

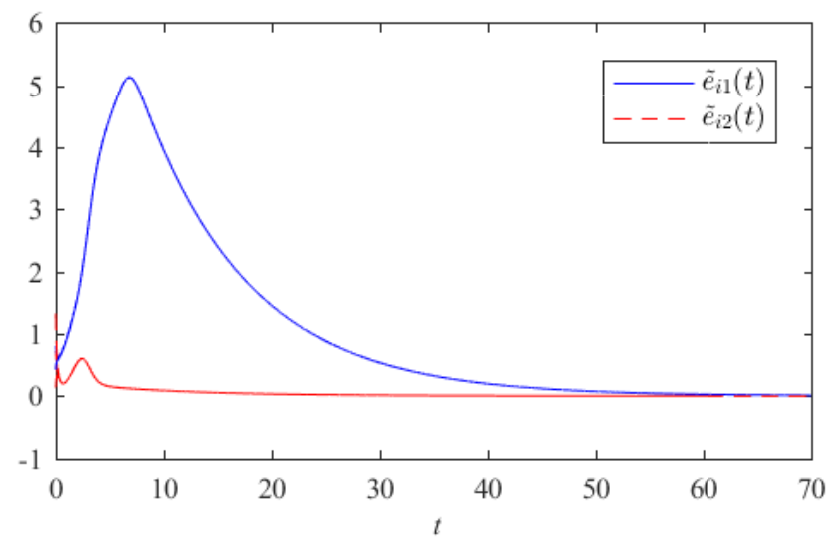

Figure 5. $\tilde{e}_{i}(t)$ with the control gains $d=2.1$ and $k=0.1$ in Example 2. 

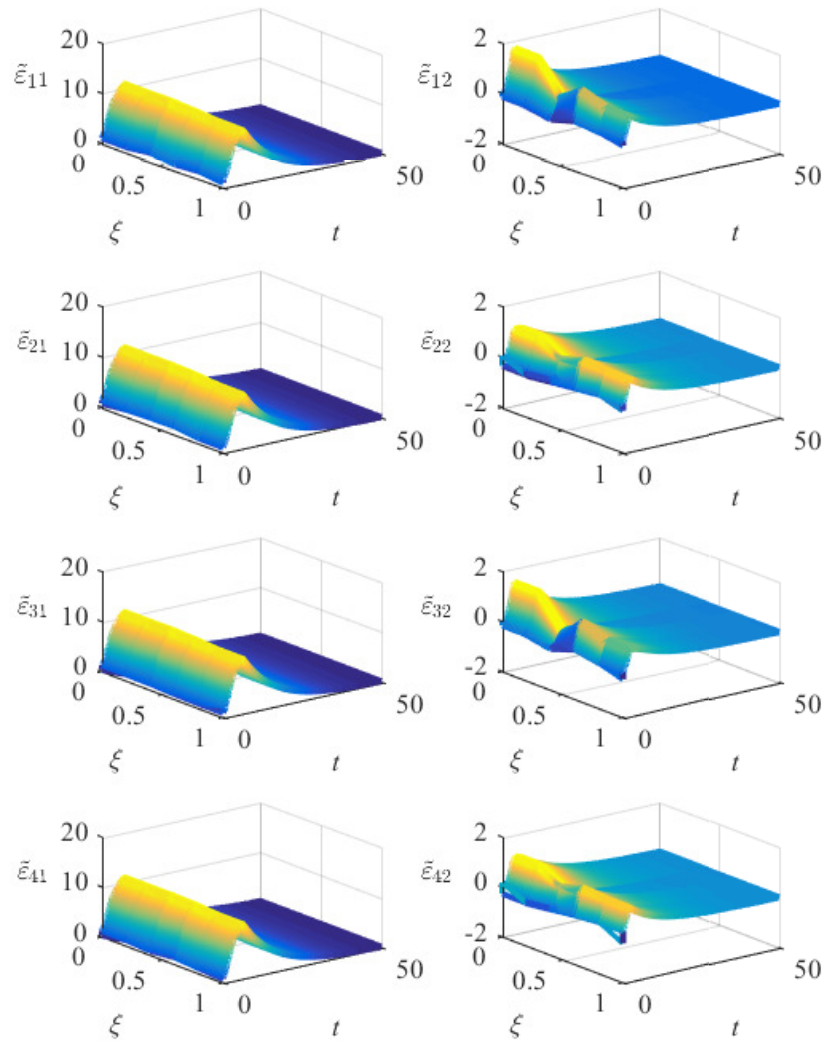

Figure 6. $\tilde{\varepsilon}_{i}(\xi, t)$ with the control gains $d=2.1$ and $k=0.1$ in Example 2.

From another point of view, $d=1.9$ and $k=0$ do not satisfy (28). It can be seen in Figures 7 and 8 that the leader-following PDE-ODEMAS cannot achieve consensus with control gains $d=0.49$ and $k=0$.

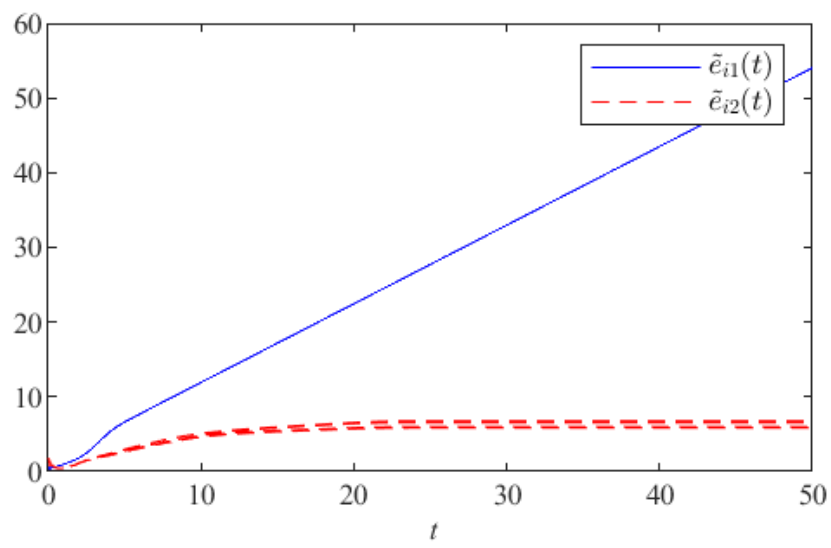

Figure 7. $\tilde{e}_{i}(t)$ with the control gains $d=1.9$ and $k=0$ in Example 2. 

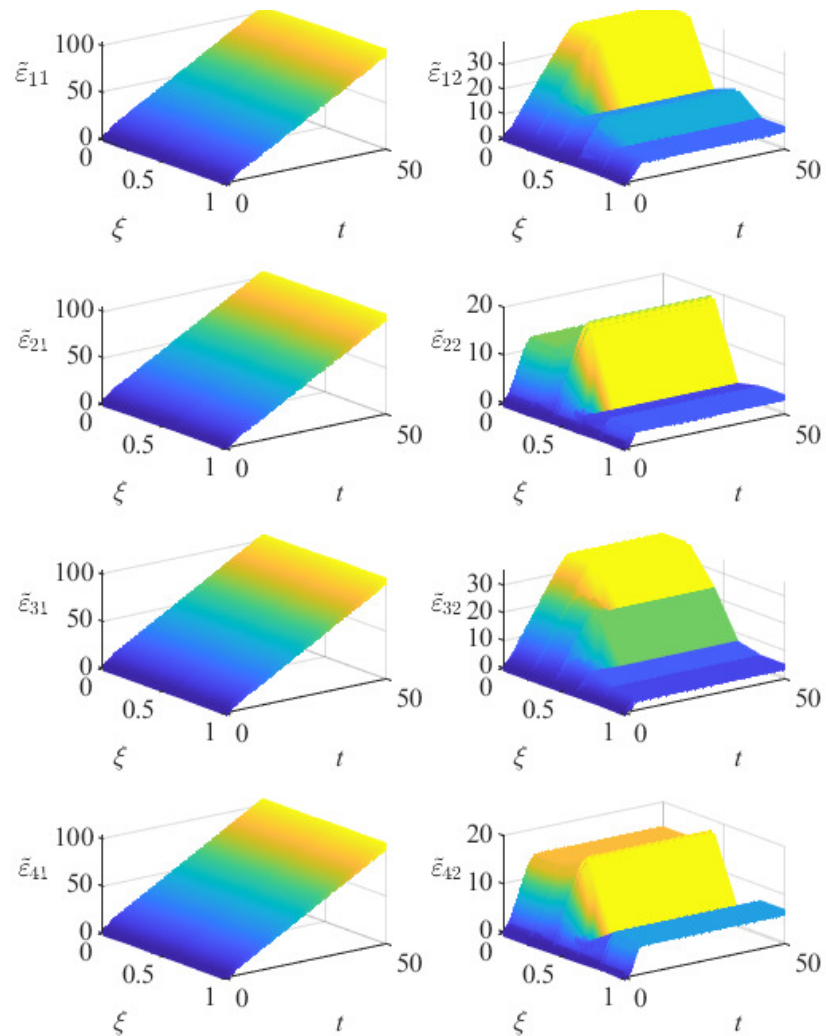

Figure 8. $\tilde{\varepsilon}_{i}(\xi, t)$ with control gains $d=1.9$ and $k=0$ in Example 2.

\section{Conclusions}

This paper has studied consensus control of the PDE-ODEMASs. First, a consensus controller of the leaderless PDE-ODEMASs was designed. We have shown that the cluster consensus behavior can be reached for the given coupling strengths for the leaderless PDE-ODEMASs. Then, a consensus controller in the leader-following PDE-ODEMASs was designed. Leader-following consensus behavior can be arrived at for the given coupling strengths for the leader-following PDE-ODEMASs. In numerical simulations, it shows the obtained gains according to the proposed methods can ensure consensus of both leaderless and leader-following PDE-ODEMASs. On the contrary, the control with gains a little bit less than those according to the proposed methods cannot achieve consensus. There are often a great number of agents in the real world and, in future, pinning consensus, only controlling a few agents of the PDE-ODEMASs, will be studied, as well as time delays.

Author Contributions: Methodology, A.Z.; software, K.Y. and Y.J.; writing-original draft preparation, X.N.; writing-review and editing, C.Y. All authors have read and agreed to the published version of the manuscript.

Funding: This research was funded by the Major Science and Technology Special Project of Yunnan Province under grant number 202102AD080002, by the Natural Science Foundation of Shandong Province under grant number ZR2019YQ28, the Development Plan of Youth Innovation Team of Universities in Shandong Province under grant number 2019KJN007.

Institutional Review Board Statement: Not applicable.

Informed Consent Statement: Not applicable.

Data Availability Statement: Not applicable.

Conflicts of Interest: The authors declare no conflict of interest. 


\section{References}

1. Mo, L.; Yuan, X.; Yu, Y. Neuro-adaptive leaderless consensus of fractional-order multi-agent systems. Neurocomputing 2019, 339, 17-25. [CrossRef]

2. Benediktsson, J.A.; Swain, P.H. Consensus theoretic classification methods. IEEE Trans. Syst. Man Cybern. 1992, 22, 688-704. [CrossRef]

3. Yang, Y.; Ji, Z.; Tian, L.; Ma, H.; Qi, Q. Bipartite consensus of edge dynamics on coopetition multi-agent systems. Sci. China Inf. Sci. 2019, 62, 229201

4. Tian, B.; Lu, H.; Zuo, Z.; Yang, W. Fixed-time leader-follower output feedback consensus for second-order multiagent systems. IEEE Trans. Cybern. 2018, 49, 1545-1550. [CrossRef]

5. Ning, B.; Han, Q.L. Prescribed finite-time consensus tracking for multiagent systems with nonholonomic chained-form dynamics IEEE Trans. Autom. Control 2018, 64, 1686-1693. [CrossRef]

6. Proskurnikov, A.V.; Matveev, A.S.; Cao, M. Opinion dynamics in social networks with hostile camps: Consensus vs. polarization. IEEE Trans. Autom. Control 2015, 61, 1524-1536. [CrossRef]

7. Du, H.; Zhu, W.; Wen, G.; Duan, Z.; Lu, J. Distributed formation control of multiple quadrotor aircraft based on nonsmooth consensus algorithms. IEEE Trans. Cybern. 2017, 49, 342-353. [CrossRef]

8. Cheng, Y.; Jia, R.; Du, H.; Wen, G.; Zhu, W. Robust finite-time consensus formation control for multiple nonholonomic wheeled mobile robots via output feedback. Int. J. Robust Nonlinear Control 2018, 28, 2082-2096. [CrossRef]

9. Shi, S.; Xu, S.; Feng, H. Robust fixed-time consensus tracking control of high-order multiple nonholonomic systems. IEEE Trans. Syst. Man Cybern. Syst. 2019, 51, 1869-1880. [CrossRef]

10. Zheng, Y.; Song, Y.; Hill, D.J.; Zhang, Y. Multiagent system based microgrid energy management via asynchronous consensus admm. IEEE Trans. Energy Convers. 2018, 33, 886-888. [CrossRef]

11. Kušić, K.; Ivanjko, E.; Vrbanić, F.; Greguric, M.; Dusparic, I. Spatial-Temporal Traffic Flow Control on Motorways Using Distributed Multi-Agent Reinforcement Learning. Mathematics 2021, 9, 3081. [CrossRef]

12. Nowzari, C.; Garcia, E.; Cortés, J. Event-triggered communication and control of networked systems for multi-agent consensus. Automatica 2019, 105, 1-27. [CrossRef]

13. Zong, X.; Li, T.; Zhang, J.F. Consensus conditions of continuous-time multi-agent systems with time-delays and measurement noises. Automatica 2019, 99, 412-419. [CrossRef]

14. Tan, X.; Cao, J.; Rutkowski, L.; Lu, G. Distributed dynamic self-triggered impulsive control for consensus networks: The case of impulse gain with normal distribution. IEEE Trans. Cybern. 2021, 51, 624-634. [CrossRef] [PubMed]

15. Liu, M.; Dong, S.; Chen, G.; Liu, M.; Wu, Z. Cooperative neural-adaptive fault-tolerant output regulation for heterogeneous nonlinear uncertain multiagent systems with disturbance. Sci. China Inf. Sci. 2021, 64, 172212.

16. Li, X.; Tang, Y.; Karimi, H.R. Consensus of multi-agent systems via fully distributed event-triggered control. Automatica 2020, 116, 108898. [CrossRef]

17. Li, X.; Yu, Z.; Li, Z.; Wu, N. Group consensus via pinning control for a class of heterogeneous multi-agent systems with input constraints. Inf. Sci. 2021, 542, 247-262. [CrossRef]

18. De la Sen, M.; Alonso-Quesada, S. On finite-time consensus objectives in time-varying interconnected discrete linear dynamic systems under internal and external delays. Adv. Mech. Eng. 2018, 10, 1687814018784840. [CrossRef]

19. Olfati-Saber, R.; Fax, J.A.; Murray, R.M. Consensus and cooperation in networked multi-agent systems. Proc. IEEE 2007, 95, 215-233. [CrossRef]

20. Aman, B.; Ciobanu, G. Knowledge dynamics and behavioural equivalences in multi-agent systems. Mathematics 2021, 9, 2869. [CrossRef]

21. Wang, J.W.; Wang, J.M. Mixed $H_{2} / H_{\infty}$ sampled-data output feedback control design for a semi-linear parabolic PDE in the sense of spatial Lo norm. Automatica 2019, 103, 282-293. [CrossRef]

22. Chen, P.; Villa, U.; Ghattas, O. Taylor approximation and variance reduction for pde-constrained optimal control under uncertainty. J. Comput. Phys. 2019, 385, 163-186. [CrossRef]

23. Chen, S.; Vazquez, R.; Krstic, M. Folding backstepping approach to parabolic PDE bilateral boundary control. IFAC-PapersOnLine 2019, 52, 76-81. [CrossRef]

24. Buyukkocak, A.T.; Aksaray, D.; Yazcoglu, Y. Planning of heterogeneous multi-agent systems under signal temporal logic specifications with integral predicates. IEEE Robot. Autom. Lett. 2021, 6, 1375-1382. [CrossRef]

25. Wang, G.; Huang, Y.; Wang, X. Consensus of semi-markov multi-agent systems with stochastically unmatched topologies. IET Control Theory Appl. 2021, 15, 1003-1017. [CrossRef]

26. d'Andréa-Novel, B.; Coron, J.M. Exponential stabilization of an overhead crane with flexible cable via a back-stepping approach. Automatica 2000, 36, 587-593. [CrossRef]

27. Hiruko, K.; Okabe, S. Controllability of hybrid PDE-ODE systems with structural instability and applications to mathematical models on intermittent hormonal therapy for prostate cancer. Math. Methods Appl. Sci. 2018, 41, 8229-8247. [CrossRef]

28. Lattanzio, C.; Maurizi, A.; Piccoli, B. Moving bottlenecks in car traffic flow: A PDE-ODE coupled model. SIAM J. Math. Anal. 2011, 43, 50-67. [CrossRef]

29. Wang, J.W.; Wu, H.N. Exponentially stabilizing fuzzy controller design for a nonlinear ODE-beam cascaded system and its application to flexible air-breathing hypersonic vehicle. Fuzzy Sets Syst. 2020, 385, 127-147. [CrossRef] 
30. Meglio, F.D.; Argomedo, F.B.; Hu, L.; Krstic, M. Stabilization of coupled linear heterodirectional hyperbolic PDE-ODE systems. Automatica 2018, 87, 281-289.

31. Zhao, Y.; Gao, H.; Qiu, J. Fuzzy observer based control for nonlinear coupled hyperbolic PDE-ODE systems. IEEE Trans. Fuzzy Syst. 2019, 27, 1332-1346. [CrossRef]

32. He, P. Consensus of uncertain parabolic PDE agents via adaptive unit-vector control scheme. IET Control Theory Appl. 2018, 12, 2488-2494. [CrossRef]

33. Fu, Q.; Du, L.; Xu, G.; Wu, J.; Yu, P. Consensus control for multi-agent systems with distributed parameter models. Neurocomputing 2018, 25, 58-64. [CrossRef]

34. Dai, X.; Wang, C.; Tian, S.; Huang, Q. Consensus control via iterative learning for distributed parameter models multi-agent systems with time-delay. J. Frankl. Inst. 2019, 356, 5240-5259. [CrossRef]

35. Fu, Q.; Du, L.; Xu, G.; Wu, J. Consensus control for multi-agent systems with distributed parameter models via iterative learning algorithm. J. Frankl. Inst. 2018, 355, 4453-4472. [CrossRef]

36. Lan, Y.-H.; Wu, B.; Shiy, Y.-X.; Luo, Y.-P. Iterative learning based consensus control for distributed parameter multi-agent systems with time-delay. Neurocomputing 2019, 357, 77-85. [CrossRef]

37. Qi, J.; Wang, S.; Fang, J.-A.; Diagne, M. Control of multi-agent systems with input delay via PDE-based method. Automatica 2019, 106, 91-100. [CrossRef]

38. Yang, C.; He, H.; Huang, T.; Zhang, A.; Qiu, J.; Cao, J.; Li, X. Consensus for non-linear multi-agent systems modelled by PDEs based on spatial boundary communication. IET Control Theory Appl. 2017, 11, 3196-3200. [CrossRef]

39. Yang, C.; Huang, T.; Zhang, A.; Qiu, J.; Cao, J.; Alsaadi, F.E. Output consensus of multiagent systems based on PDEs with input constraint: A boundary control approach. IEEE Trans. Syst. Man Cybern. Syst. 2021, 51, 370-377. [CrossRef]

40. Seuret, A.; Gouaisbaut, F. Jensen's and Wirtinger's inequalities for time-delay systems. IFAC Proc. Vol. 2013, 46, 343-348. [CrossRef]

41. Pilloni, A.; Pisano, A.; Orlov, Y.; Usai, E. Consensus-based control for a network of diffusion PDEs with boundary local interaction. IEEE Trans. Autom. Control 2015, 61, 2708-2713. [CrossRef]

42. Wang, J.W.; Krstic, M. Output-feedback boundary control of a heat PDE sandwiched between two ODEs. IEEE TRansactions Autom. Control 2019, 64, 4653-4660. [CrossRef] 